\title{
Solitary fibrous tumor
}

INSERM

\section{Source}

INSERM. (1999). Orphanet: an online rare disease and orphan drug data base. Solitary fibrous tumor. ORPHA:2126

Solitary fibrous tumor (SFT) represents a diverse group of ubiquitous rare spindle cell neoplasms that may be benign or malignant and that most frequently arises from the pleura and peritoneum and rarely from other sites such as head and neck, liver and skeletal muscle. SFT may be clinically asymptomatic or may present with enlarging mass, compressive effects depending on the site involved and rarely with paraneoplastic manifestations (osteoarthropathy or hypoglycemia). 